\title{
Narrativa
}

narrativa

Nuova serie

43 | 2021

La fantascienza nelle narrazioni italiane ipercontemporanee

\section{David FORGACS, Messaggi di sangue. La violenza nella storia d'Italia}

\section{Cecilia Ridani}

\section{(2) OpenEdition \\ Sournals}

Edizione digitale

URL: https://journals.openedition.org/narrativa/478

DOI: $10.4000 /$ narrativa.478

ISSN: 2804-1224

Editore

Presses universitaires de Paris Nanterre

\section{Edizione cartacea}

Data di pubblicazione: 1 décembre 2021

Paginazione: $297-298$

ISBN: 978-2-84016-485-2

ISSN: $1166-3243$

\section{Notizia bibliografica digitale}

Cecilia Ridani, «David forgacs, Messaggi di sangue. La violenza nella storia d'Italia», Narrativa [Online], 43 | 2021, online dal 01 novembre 2021, consultato il 03 novembre 2022. URL: http://

journals.openedition.org/narrativa/478 ; DOI: https://doi.org/10.4000/narrativa.478

Questo documento è stato generato automaticamente il 3 novembre 2022.

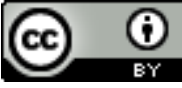

Creative Commons - Attribuzione 4.0 Internazionale - CC BY 4.0

https://creativecommons.org/licenses/by/4.0/ 


\title{
David FORGACS, Messaggi di sangue. La violenza nella storia d'Italia
}

\author{
Cecilia Ridani
}

\section{NOTIZIA}

David fORGACS, Messaggi di sangue. La violenza nella storia d'Italia, Bari, Laterza, 2020, 377 p.

1 In che modo i mezzi di comunicazione hanno trasmesso, distorto o censurato le informazioni sulle atrocità che hanno segnato la storia dell'Italia contemporanea? Qual è il rapporto tra atto violento e funzione comunicativa? Cosa ha lasciato impresso la violenza nella memoria collettiva?

2 A questi interrogativi risponde David Forgacs nel suo ultimo libro che, sin dal titolo invita a rileggere la storia italiana come una sequenza di messaggi di sangue. Riconoscendo la complessità e l'instabilità dell'oggetto violenza, nonché il suo carattere proteiforme, l'autore consacra ognuno dei dodici capitoli che compongono il volume ad uno o più atti coercitivi che si sono compiuti nella Penisola e nelle sue colonie dal Risorgimento a oggi. L'indagine di Forgacs privilegia una prospettiva diacronica che consente da una parte di cogliere le linee di continuità e di discontinuità nelle modalità di comunicazione della violenza, dall'altra di esplorare il legame che essa intrattiene con il potere, da cui talvolta viene coperta o legittimata. Il libro, arricchito da un supporto iconografico di notevole impatto visivo, si apre con una disamina sui processi comunicativi degli atti di ferocia nel contesto delle lotte risorgimentali. Si passano poi in rassegna alcune delle efferatezze che hanno scandito i primi anni del Novecento e che sono state distorte o censurate dalla stampa nazionale e dalle istituzioni, come la strage di civili arabi in Libia nel 1911 e le esecuzioni dei soldati italiani durante il primo conflitto mondiale. Segue lo studio di memoriali e diari di ex squadristi fascisti, in cui la violenza trova giustificazione nella difesa patriottica contro il bolscevismo o come punizione per i socialisti, per poi scandagliare, nel capitolo successivo un altro caso di atrocità negata, quale il massacro degli etiopi per mano delle milizie fasciste nel 1937. 
3 Per chi subisce un'aggressione non è facile denunciare, come dimostrano le pagine dedicate agli stupri nel Basso Lazio, mentre nel capitolo consacrato alla lotta partigiana prende forma una meditazione sul conflitto interpretativo a cui può dare origine un atto di ferocia e che, talvolta, rovescerebbe il senso stesso della morte. I messaggi scritti con il sangue delle vittime del terrorismo "nero" e "rosso" e della mafia diventano infine, un'occasione per ripensare le dimensioni individuali, sociali e politiche della violenza.

Il volume si chiude con un'analisi sui moderni canali di informazione, i quali oggigiorno rivestono un ruolo fondamentale nella propagazione della brutalità; a tal riguardo vengono presi in esame due episodi di cronaca recente, gli scontri del G8 di Genova e l'odio razziale perpetuatasi nel 2011 - in ben due circostanze - a opera di cittadini italiani contro immigrati.

5 In un libro trascinante e denso di riflessioni, Forgacs pratica un'indagine sull'Italia liberale e repubblicana, mostrando come la violenza si insinui nelle fessure della sua storia, diventando essa stessa una forma di comunicazione. 